\title{
Optimizing allocation in a warehouse network
}

\author{
Malaguti, Enrico \\ enrico.malaguti@unibo.it
}

\author{
Nannicini, Giacomo \\ nannicini@us.ibm.com
}

Thomopulos, Dimitri

dimitri.thomopulos@unibo.it

\begin{abstract}
We study the problem faced by an international retail company operating e-commerce to determine the initial allocation of goods into warehouses, so as to provide customers in local markets with high level of service. We represent the movement of goods through the warehouse network to the customers using linear and quadratic integer programming models.
\end{abstract}

\section{Introduction}

We consider an international retail company operating e-commerce on a global scale. The retailer sells on local markets thorough dedicated websites, where customers can order available goods from an online catalog. Ordered goods are then shipped to customers by express delivery service. The retailer manages a network of warehouses consisting of a main warehouse in the European Union and other warehouses outside Europe, each one associated with a regional market. European customers are always served from the main warehouse, while non-European customers can be served from the local warehouses or from the central warehouse. Hence, even after an initial allocation of goods to the central and regional warehouses, there can be a continuos flow of goods from the central and local warehouses to the customers. For a potential order, the origin and destination of the shipment define different levels of service. A shipment from a local warehouse is compatible with fast shipping and is associated with better level of service, while an intercontinental shipping requires longer time. We study the problem that the retail company faces when deciding the initial allocation of goods into warehouses. On the one hand, the company can store items at local warehouses, thus maximizing the level of service and minimizing shipping cost, although the initial acquisition of goods at local warehouses has (on average) larger cost. In addition, items cannot travel from the local to the main (European) warehouse, implying that an item allocated at a local warehouse cannot be sold on a different market. On the other hand, 
the retailer can store items in the central warehouse, having more flexibility (each item can be sold to any local market, and at the end of the season unsold items are already consolidated in a single location to be salvaged) and smaller acquisition cost for the initial stock. Flexibility is obtained at the cost of providing a lower level of service, potentially losing a share of the sales, and having a larger shipping cost.

We model and study the problem for increasing levels of detail. We consider a multi-period planning problem, and we initially assume that the demand and price of goods are given for each period. In this case, the problem is decomposable and solvable by inspection if no constraint that links the different item categories (e.g., capacity or assortment constraints at the warehouses) is imposed. Then, we consider the case in which prices can be set in each period, and the demand is determined accordingly. In particular, we study the case of discrete price levels, each one associated with an expected demand.

The problem we consider has the structure of classical location-allocation problems, but it is also an inventory problem with elements of network flows, and pricing. The literature on location-allocation problems is vast, including textbooks (see [3]) and several review papers, some of which are quite recent (e.g., [1]). Two main elements characterize our paper and its motivating application: first, our models incorporate explicitly the sensitivity of the demand to the level of service, which is not a common feature (see $[2])$; second, the models incorporate pricing decisions and return of purchased items: both elements may substantially change the attractiveness of a candidate location. Papers integrating location and inventory decisions are not very common; an example is [5], where a location-inventory problem involving a single supplier and multiple retailers with variable demand is studied.

In the next section we formally define the allocation and flow problems we study, while in Section 3 we present some computational experiments based on real data from the retailer. Concluding remarks and future work are presented in Section 4.

\section{Mathematical models}

We consider a multi-period planning problem with $T$ periods (indexed by $t=0, \ldots, T)$. The retail company wants to decide the initial allocation (period 0 ) of $m$ item categories, indexed by $i=1, \ldots, m$, where the total initial amount $u_{i}^{0}$ of each category (to be shared among the warehouses) is the result of merchandising decisions and is given. The company manages $n$ warehouses, indexed by $j=1, \ldots, n$, and associated with $n$ markets, where $j=1$ is the main warehouse.

Items are sold on a local market associated with each warehouse. Item 
$i$ can be allocated to warehouse $j$ at an initial cost $\alpha_{i j}$, and can be moved from the main warehouse 1 to a warehouse $j>1$, in order to satisfy a local demand at $j$ for an item category which is not available at the local warehouse. Items can be moved at any time during each period by paying a (strictly positive) cost $\zeta_{i j}$, representing intercontinental shipping and import taxes. The models that we develop incorporate the sensitivity of the demand to the level of service (shipping time). If an item to be sold on market $j$ during period $t$ is not available at the local warehouse and has to be shipped from the main warehouse, the demand is reduced by a fraction $\beta$, representing the share of lost orders. In addition, the models consider item returns: a specified fraction $\rho$ of the orders of each market is returned to the local warehouse with a delay of $\tau$ time periods. Finally, we consider generic constraints defining required initial assortment and capacity constraints at the warehouses. These are the only constraints that link the different item categories.

Fixed Price-Demand In our first model we assume the demand $\delta_{i j}^{t}$ and price $\pi_{i j}^{t}$ for each good $i$, warehouse $j$ and time period $t$ are given. Assuming the entirety of the demand has to be satisfied, the problem can be sen as a cost minimization problem: the total revenue is constant, and we have to define an initial allocation in such a way that the overall acquisition and relocation cost, plus the value of lost orders, is minimized.

We model the problem as a Mixed-Integer Linear Program (MILP), although special cases (discussed later) can be solved in closed form. We use variables $w_{i j}^{t}$ to define the level of goods of type $i$ at warehouse $j$ at period $t$ (for these these variables, integrality is imposed only at period 0 , which represents the initial allocation), integer variables $y_{i j}^{t}$ to define the quantity of goods of type $i$ moved from the central warehouse 1 to local warehouse $j$ at period $t$ and variables $z_{i j}^{t}$ to define the amount of lost sales of goods of type $i$ at market $j$ at period $t$. Our MILP model is as follows: 


$$
\begin{array}{rr}
\min \sum_{i=1}^{m} \sum_{j=1}^{n}\left(\alpha_{i j}^{0} w_{i j}^{0}-\alpha_{i j}^{T} w_{i j}^{T}\right)+\sum_{t=1}^{T} \sum_{i=1}^{m} \sum_{j=2}^{n}\left(\zeta_{i j}^{t} y_{i j}^{t}+\pi_{i j}^{t} z_{i j}^{t}\right) & (1) \\
w_{i j}^{t}=w_{i j}^{t-1}-d_{i j}^{t}+y_{i j}^{t}+z_{i j}^{t}+\rho_{j}\left(\delta_{i j}^{t-\tau}-z_{i j}^{t-\tau}\right) & \forall i, j>1, t>\emptyset 2) \\
w_{i 1}^{t}=w_{i 1}^{t-1}-d_{i 1}^{t}-\sum_{j=2}^{n} y_{i j}^{t}+\rho_{j} \delta_{i 1}^{t-\tau} & \forall i, t>0 \\
z_{i j}^{t}=\beta\left(y_{i j}^{t}+z_{i j}^{t}\right) & \forall i, t>0, j>(3) \\
f\left(w_{j}^{0}\right) \leq 0 & \forall j \\
w_{i j}^{0} \geq 0 \text { integer } & \forall i, j \\
y_{i j}^{t} \geq 0 \text { integer } & \forall i, j>1, t>(6) \\
w_{i j}^{t} \geq 0 & \forall i, j, t>0 \\
z_{i j}^{t} \geq 0 & \forall i, j>1, t>(8)
\end{array}
$$

where the objective function (1) minimizes the initial allocation (minus salvage value) and relocation costs, and the value of lost orders. Constraints (2) and (3) define balance for the local and central warehouses, respectively; and constraints (4) define the share of lost sales depending on the level of service of each order. Constraints (5), where $f($.$) is any linear function,$ are relative to the initial allocation of items to each warehouse $j$, and can be used, e.g., to model capacity constraints at the warehouses, or initial assortment requirements.

Proposition 1. Model (1) - (4), (6) - (9) is decomposable for each good type $i=1, \ldots, m$, and each warehouse $j=1, \ldots, n$, and solvable by inspection.

Proof. If constraints (5) are not imposed, no constraint links the different goods. Similarly, there is no constraint linking local warehouses. For a given item category $i$, the only decision to be taken is about the initial quantity $w_{i j}^{0}$ of $i$ to be stored at the local warehouse $j$ to serve market $j$. For each value, all other model variables and hence the objective function can be computed in closed form.

Demand as discrete function of price Next, we consider the more involved case where the demand is not given and it is a function of discrete price values. This allows us to model a discount policy during the time horizon. We model three levels of price and demand through binary variables $x_{i j l}^{t}$ indicating if item category $i$ in period $t$ is sold from warehouse $j$ at price $\pi_{i j l}$. In this case, we expect a demand $\delta_{i j l}$. Prices are defined so as $\pi_{i j 1}>\pi_{i j 2}>\pi_{i j 2}, \forall i, j$. We denote by $s_{i j}^{t}$ the number of items $i$ sold from warehouse $j$ during period $t$. We can formulate a Mixed-Integer Quadratic Program (MIQP) as follows: 


$$
\begin{aligned}
& \max \sum_{t=1}^{T} \sum_{i=1}^{m} \sum_{j=1}^{n}\left(1-\rho_{j}\right) s_{i j}^{t} \sum_{l=1}^{3} \pi_{i j l} x_{i j l}^{t} \\
& -\sum_{i=1}^{m} \sum_{j=1}^{n}\left(\alpha_{i j}^{0} w_{i j}^{0}-\alpha_{i j}^{T} w_{i j}^{T}\right)-\sum_{t=1}^{T} \sum_{i=1}^{m} \sum_{j=2}^{n} \zeta_{i j}^{t} y_{i j}^{t} \\
& w_{i j}^{t}=w_{i j}^{t-1}-s_{i j}^{t}+y_{i j}^{t}+\rho_{j} s_{i j}^{t-\tau} \quad \forall i, j>1, t>0 \\
& w_{i 1}^{t}=w_{i 1}^{t-1}-s_{i 1}^{t}-\sum_{j=2}^{n} y_{i j}^{t}+\rho_{j} s_{i 1}^{t-\tau} \quad \forall i, t>0 \\
& s_{i 1}^{t} \leq \sum_{l=1}^{3} \delta_{i 1 l}^{t} x_{i 1 l}^{t} \quad \forall i, t>0 \\
& s_{i j}^{t} \leq \sum_{l=1}^{3} \delta_{i j l}^{t} x_{i j l}^{t}-\frac{\beta}{1-\beta} y_{i j}^{t} \quad \forall i, j>1, t>0 \\
& x_{i j 1}^{t} \leq x_{i j 1}^{t-1} \quad \forall i, j, t>1 \\
& x_{i j 2}^{t} \leq x_{i j 1}^{t-1}+x_{i j 2}^{t-1} \quad \forall i, j, t>1 \\
& x_{i j 1}^{t}+x_{i j 2}^{t}+x_{i j 3}^{t}=1 \quad \forall i, j, t>0 \\
& \sum_{j=1}^{n} w_{i j}^{0} \leq u_{i}^{0} \quad \forall i \\
& f\left(w_{j}^{0}\right) \leq 0 \quad \forall j \\
& w_{i j}^{0} \geq 0 \text { integer } \quad \forall i, j \\
& y_{i j}^{t} \geq 0 \text { integer } \quad \forall i, j>1, t>0 \\
& s_{i j}^{t} \geq 0 \text { integer } \quad \forall i, j, t>0 \\
& w_{i j}^{t} \geq 0 \quad \forall i, j, t>0 \\
& x_{i j l}^{t} \in\{0,1\} \quad \forall i, j, l, t>0
\end{aligned}
$$

where the objective function (10) is a quadratic function that maximizes the sales profit, minus initial allocation and relocation cost; constraints (11) and (12) define the goods balance for the local and central warehouses, respectively; constraints (13) and (14) define the demand function; constraints (15) and (16) impose that price is nonincreasing; finally we use type 1 SOS constraints (17) to impose uniqueness of the selected level of demand and price. Again, (19) can be any linear constraints linking the variables for the initial allocation of items at each warehouse $j$.

In order to linearize model (11)-(24), we replace the first term of the 
objective function by

$$
\max \sum_{t=1}^{T} \sum_{i=1}^{m} \sum_{j=1}^{n}\left(1-\rho_{j}\right) p_{i j}^{t}
$$

and add the following constraints

$$
\begin{array}{rc}
p_{i j}^{t} \leq \pi_{i j} s_{i j}^{t} & \forall i, j, t>0 \\
p_{i j}^{t} \leq \pi_{i j l} s_{i j}^{t}+M\left(1-x_{i j l}^{t}\right) & \forall i, j, t>0, l=2,3
\end{array}
$$

where $M$ is a suited large constant which inactivates the constraint when the associated $x$ variables is 0 . The following proposition states the effect of removing linking constraints (19) from the model.

Proposition 2. Model (10) - (18), (20) - (24) is decomposable for each item category $i=1, \ldots, m$.

\section{Computational experiments}

We report experiments performed on real data from the retailer. We linearize model (10)-(24) as explained in the previous section and solve it with the general purpose MILP solver of CPLEX 12.6. All experiments are performed on a single core of a Core2 Quad Q9300 $2.50 \mathrm{GHz}$ computer with 8 GB RAM, running Linux.

In the reported experiments we consider a time horizon of six month divided into periods of one month. In Table 1 we consider instances of the model without linking constraints (19); hence, the problem is decomposable and solved separately for each item category. We report results from six different instances, and we consider two values for $\beta$, the share of lost sales at local markets when the shipment originates from the central warehouse (sensitivity to level of service). All instances are solved in few seconds, and the table reports, after the instance name, the number of Branch-andBound nodes explored by the MILP solver, the computing time in seconds, the MILP size (number of variables and constraints) and the value of the parameter $\beta$. Despite the fact that the problems are quite small, they are far from trivial for the solver, which has to explore several hundred nodes to find the optimal solution.

Then, we consider the more difficult problem arising when goods are linked by constraints of type (19), and the model in not separable. In these experiments we consider a single knapsack constraint for each warehouses at time 0 . In Table 2 we consider three instances having an increasing number of item categories, namely, 10, 25 and 50. In addition to this number and information in common with the previous table, we also report optimality gaps after two hours of computation, and the computing time. Unsurprisingly, since the problem is no longer separable, Table 2 confirms that the 
Figure 1: Assignment of goods to the central warehouse as a function of $\beta$.

Figure 2: Assignment of goods to the central warehouse as a function of transportion costs (including taxes).

models become significantly more challenging. All instances hit the time limit, with the exception of the smallest one with $\beta=0.5$, but the final optimality gaps do not exceed $6 \%$.

Figure 1 represents the initial allocation of a single good at the central warehouse for increasing values of the parameter $\beta$, i.e. the sensitivity of customers to the level of service (shipping delay). The figure considers the six instances of table 1 . For increasing values of $\beta$, we observe that the initial allocation of items to the central warehouse is reduced (i.e., goods are allocated to the regional warehouses, close to their destination markets). The curves become flat when the central warehouse only contains items to be sold on its associated market.

A similar behavior is observed in Figure 2, where we represent the initial allocation of goods at the central warehouse for different values of the shipping and tax cost. The horizontal axis of the figure reports the value of a coefficient multiplying the actual costs ( $\zeta$ in equation (10)). For increasing values of the costs, we observe that the initial allocation to the central warehouse is reduced. Indeed, it is less profitable to keep goods at the central warehouse when they have to be shipped to a local market.

\section{Conclusions}

We considered the allocation and pricing problem faced by an international retail company operating e-commerce, when serving customers in markets that are sensitive to level of service. We developed decision models of increasing complexity, and tested these models on instances derived from real data. The experiments showed that the problems are challenging, justifying the future development of heuristic approaches and decomposition methods for solving large-scale instances. In addition, the real application has several stochastic features, and we plan to extend the analysis presented in this paper in order to incorporate stochasticity (see, e.g., [4]) into our optimization models.

\section{References}

[1] Melo, M. T. and S. Nickel, and F. Saldanha-da-Gama, Facility location and supply chain management: A review, European Journal of Operational Research, 196 (2008) 401-412. 


\begin{tabular}{lrrrrrr}
\hline Instance & B\&B nodes & Time & Variables & Constraints & $\beta$ & Initial Assign. \\
\hline SG1 & 1362 & 0.6 & 228 & 264 & 0.2 & 47 \\
SG1 & 1571 & 0.3 & 228 & 264 & 0.5 & 28 \\
SG2 & 533 & 0.4 & 228 & 264 & 0.2 & 60 \\
SG2 & 940 & 0.4 & 228 & 264 & 0.5 & 40 \\
SG3 & 1197 & 0.8 & 228 & 264 & 0.2 & 23 \\
SG3 & 652 & 0.8 & 228 & 264 & 0.5 & 12 \\
SG4 & 1135 & 0.8 & 228 & 268 & 0.2 & 66 \\
$S G 4$ & 1010 & 0.3 & 228 & 268 & 0.5 & 58 \\
$S G 5$ & 970 & 0.5 & 228 & 268 & 0.2 & 63 \\
$S G 5$ & 940 & 0.5 & 228 & 268 & 0.5 & 45 \\
$S G 6$ & 14283 & 5.2 & 228 & 268 & 0.2 & 63 \\
$S G 6$ & 1398 & 0.6 & 228 & 268 & 0.5 & 54 \\
\hline
\end{tabular}

Table 1: Computing performance for instances of a single good category.

\begin{tabular}{lrrrrrrr}
\hline Instance & \# good cat. & B\&B nodes & \% Gap & Time & Variables & Constraints & $\beta$ \\
\hline MG1 & 10 & 3826127 & 0.30 & T.L. & 2280 & 2644 & 0.2 \\
MG1 & 10 & 340578 & 0.00 & 500.5 & 2280 & 2644 & 0.5 \\
MG2 & 25 & 1751676 & 3.36 & T.L. & 5700 & 6604 & 0.2 \\
MG2 & 25 & 1146056 & 0.93 & T.L. & 5700 & 6604 & 0.5 \\
MG3 & 50 & 400177 & 3.73 & T.L. & 11400 & 13204 & 0.2 \\
MG3 & 50 & 886181 & 5.42 & T.L. & 11400 & 13204 & 0.5 \\
\hline
\end{tabular}

Table 2: Computing performance for instances of a multiple good categories, (time limit of 2 hours). 
[2] Klose, A. and A. Drexl, Facility location models for distribution system design, European Journal of Operational Research, 162 (2005) 4-29.

[3] Simchi-Levi, D. and P. Kaminsky, and E. Simchi-Levi, Designing and Managing the Supply Chain: Concepts, Strategies, and Case Studies, New York, McGraw-Hill/Irwin, 2008.

[4] Snyder, L.S., Facility location under uncertainty: a review, IIE Transactions, 38 (2006) 547-564.

[5] Shen, Z.-J. M., and C. Coullard, and M. S. Daskin, A Joint LocationInventory Model, Transportation Science, 37 (2003) 40-55. 